\title{
Towards Wearable and Flexible Sensors and Circuits Integration for Stress Monitoring
}

\author{
Ching-Mei Chen, Member IEEE, Salzitsa Anastasova, Member IEEE, Kai Zhang, Bruno G. Rosa, \\ Member IEEE, Benny P. L. Lo, Senior Member IEEE, Hazel E. Assender and Guang-Zhong Yang, \\ Fellow IEEE
}

\begin{abstract}
Ching-Mei Chen is with Department of Engineering Science, University of Oxford, Oxford, OX1 3PJ, UK. (E-mail:ching-mei.chen@eng.ox.ac.uk).
Kai Zhang and Hazel E. Assender, are with Department of Materials, University of Oxford, Oxford, OX1 3PJ UK. (E-mail: hazel.assender@materials.ox.ac.uk) Salzitsa Anastasova, Bruno Gil Rosa, Benny P. L. Lo and Guang-Zhong Yang are with The Hamlyn Centre, Imperial College London, London, SW7 2AZ, UK. (E-mail: \{salzitsa.anastasova, b.gil-rosa, benny.lo, g.z.yang\}@imperial.ac.uk).
\end{abstract}

\begin{abstract}
Excessive stress is one of the main causes of mental illness. Long-term exposure of stress could affect one's physiological wellbeing (such as hypertension) and psychological condition (such as depression). Multisensory information such as heart rate variability (HRV) and $\mathrm{pH}$ can provide suitable information about mental and physical stress. This paper proposes a novel approach for stress condition monitoring using disposable flexible sensors. By integrating flexible amplifiers with a commercially available flexible polyvinylidene difluoride (PVDF) mechanical deformation sensor and a pH-type chemical sensor, the proposed system can detect arterial pulses from the neck and $\mathrm{pH}$ levels from sweat located in the back of the body. The system uses organic thin film transistor (OTFT)-based signal amplification front-end circuits with modifications to accommodate the dynamic signal ranges obtained from the sensors. The OTFTs were manufactured on a low-cost flexible polyethylene naphthalate (PEN) substrate using a coater capable of Roll-to-Roll (R2R) deposition. The proposed system can capture physiological indicators and data be interrogated by Near Field Communication (NFC). The device has been successfully tested with healthy subjects, demonstrating its application for real-time stress monitoring.
\end{abstract}

Index Terms-HR, PVDF, OTFT, pH sensor, Wearable device, Flexible circuits, HRV, BPM

\section{INTRODUCTION}

T TRESS response is also known as 'fight or flight' response, which describes the body reactions due to rapid hormone changes when a person is exposed to a stressor. It was first defined by Walter Cannon in the 1920s and, ever since, extensive studies have been carried out to understand the causes and responses of human stress due to different factors.

When a person is under stress, there are a few apparent symptoms related to stress. These include changes in heart rate variability (HRV), elevated blood pressure and reduced $\mathrm{pH}$ level [1-3]. Stress disorders can be caused by long-term chronic stress, posttraumatic stress disorders, emotions and behavioral/physiological adaptions. To manage excessive stress, self-monitoring is one of the possible ways to avoid stressors. Fear-induced film clips or violent game test protocols [4-6] have been widely used to stimulate stress levels, by emulating situations where a person is exposed to some degree of danger. In most of the studies, electronic recording devices were used to record the physiological responses of the subjects. ECG signals (HRV) are found to be a reliable indicator of stress though, for reliable and robust monitoring, a multisensory approach is more desirable, as proposed in this study. For instance Rosa and Yang [7] recently presented a mental health assessment platform with ECG, galvanic skin response (GSR), temperature and bio-motion detection channels, which led the authors to achieve an $89 \%$ accuracy in the distinction between exercise (physical stress), rest and mental activities performed by healthy subjects in controllable experimental conditions. We intend to extend this study by developing new types of sensors and interfaces to ne included on stress monitoring platforms.

Wearable devices have attracted much attention for their potential use for pervasive healthcare monitoring in the past two decades [8-10]. With wireless connectivity, these devices enable low-cost, disposable, long-term and self-monitoring solutions to be deployed at home and hospital settings. However, these devices are often made with rigid printed circuit boards (PCB) and enclosures, which hinder the placement of the devices over the human body for accurate measurement of biological signals. In addition, the design of these wearable devices may not be suitable for patients with mental illnesses, e.g., a wrist worn device may not be appropriate for a patient with tendency of self-harm. Flexible electronics [11] is an emerging technology that can enhance the performance of sensor fabrication, interface and signal conditioning for wearable devices. Its low production cost could enable, on one hand, the development of disposable devices with high standard of hygiene [12] and, on the other hand, the development of wearable devices as thin and cheap as plasters, making them safer alternatives for physical stress monitoring during/after exercises and emotional stress assessment for patients with some sort of mental condition.

The aim of this research work is to design OTFT-based amplifiers for capturing bio-signals, such as artery pulses and/or ECG, 
using the piezoelectric response of PVDF [13-14], combined with $\mathrm{pH}$ level assessment in sweat [15-16]. Although these sensors and signal conditioning circuits were presented useful in the past [13-16] for healthy subjects' data collection, there aren't yet any fully integrated devices onto a common bendable substrate, suitable for mass production using low-cost manufacturing processes and equipment. Moreover, the need for flexible devices that are structurally conformal with the body shape is important to help minimizing the individual and societal stigmas associated with the use of medical devices, especially for mental health patients. Within the regard, the present manuscript proposes a wearable and flexible system for continuous mental health monitoring and produced by low-cost R2R deposition process. The output voltages of the developed amplifiers developed are connected directly to a wireless signal processing sensor node [17-18] for real-time emotional and physical stress monitoring, as shown in Fig. 1.

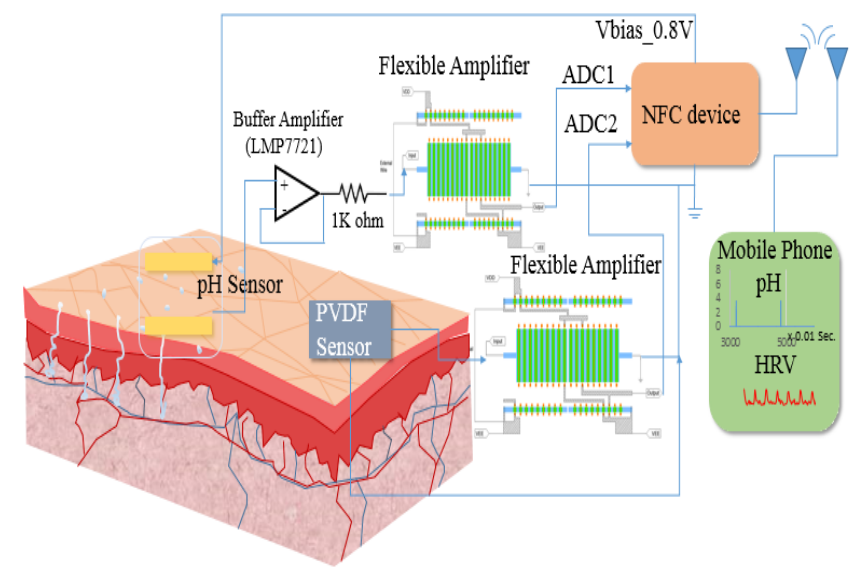

Fig. 1. Block diagram of the proposed wearable and flexible system for mental healthcare monitoring.

\section{Organic Thin Film Transistor Circuits Design, SEnSors InTEgration and Radio Frequency Data Transmission}

R2R technology is a preferred method for manufacturing low cost, lightweight and flexible devices. The amplifiers for the signal conditioning chain of the proposed system were manufactured using a R2R-based deposition process [19]. Vacuum metallization by physical vapor deposition (PVD) enables the fabrication of OTFT-based electrodes with all-vacuum printing technique. A flash evaporation technique is used to deposit polymer thin film on fast moving plastic webs, enabling fast deposition of dielectric materials for OTFT [15], which is paired with semiconductor dinaphtho[2,3-b:2',3'-f] thieno[3,2-b]thiophene (DNTT) to form devices with high reproducibility and production yield. Small molecule semiconductor materials have attracted much attention lately like DNTT that exhibits higher stability than pentacene, which can be vacuum-deposited with higher compatibility with electrodes and multilayer devices than polymers, such as poly(3-hexylthiophene-2,5-diyl) (P3HT). In addition, the extra oxygendoping effect enables relatively high mobility [20-23]. An OTFT-based prototype sheet manufactured from our R2R processing facility is shown in Figure 2. Fifty-four P-channel transistors were fabricated and grouped into 6 common gate designs, with two different widths (W1: 2200um and W10: 22000um) for development of sub-circuits.

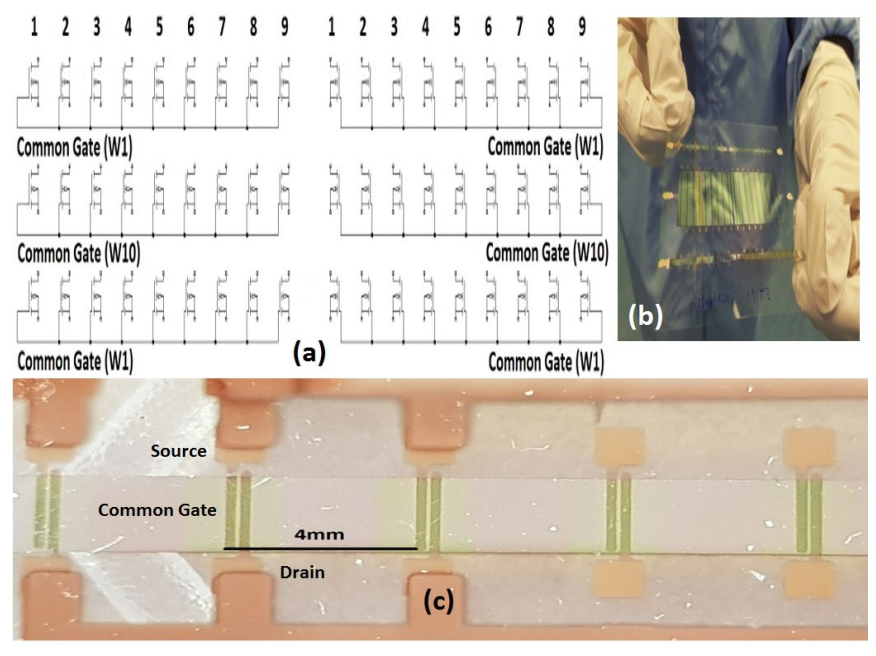

Fig. 2. (a) OTFT prototype sheet schematic (b) photo of OTFTs prototype sheets (c) A photo demonstrates W1 transistors and interconnects of a current mirror. 


\section{A. OTFT-based transimpedance amplifier design}

A simple OTFT-based transimpedance amplifier (version 1) was developed and shown in Fig. 3. M1-M3 are 1:1:1 simple current mirrors, with the current of each path $\left(\mathrm{I}_{1}, \mathrm{I}_{2}\right.$ and $\left.\mathrm{I}_{3}\right)$ being set and biased by OTFT-based diode M4 ( 200 nA). In this circuit, M5 and M6 can be regarded as current-steering devices, which feed the input signals into the gate of M5 and transistor M6 controlling the gate swing voltage of M9 and, therefore, the output voltage of the proposed OTFT-based amplifier. M7 and M8 act as 'source follower' resistors (as referred in conventional CMOS design techniques) and $\mathrm{R}_{\mathrm{f}}$ is the resistance that determines the current-tovoltage conversion gain. To enable the connection of the flexible amplifier to a radio frequency transmission chip (typically powered by $3.3 \mathrm{~V}$ ), $\mathrm{Rf}$ is fixed and validated with a value of $620 \mathrm{k} \Omega$. The rail-to-rail voltage of this amplifier is $+5 \mathrm{~V} /-5 \mathrm{~V}$, though it can be replaced by a $9 \mathrm{~V}$ battery for the purpose of a wearable device, whereas the total power consumption is $6 \mathrm{uW}$. Table 1 shows the W/L values of the transistors that were developed in the circuitry. For double width (W2) and triple width (W3) transistors, we simply used two W1 transistors and three W1 transistors in parallel, by deposition of copper interconnects to enable complete signal routing between the terminals of the aforementioned transistors.

\begin{tabular}{|c|c|c|}
\hline TABLE I & OTFTs Amplifiers Width/Length information \\
\hline \hline Transistor No. & Amplifier $1 \mathrm{~W} / \mathrm{L}$ information & Amplifier $2 \mathrm{~W} / \mathrm{L}$ information \\
\hline M1(W1) & $2500 \mathrm{um} / 115 \mathrm{um}$ & $2500 \mathrm{um} / 110 \mathrm{um}$ \\
\hline M2(W1-A1,W3-A2) & $2500 \mathrm{um} / 115 \mathrm{um}$ & $7500 \mathrm{um} / 110 \mathrm{um}$ \\
\hline M3(W1-A1,W3-A2) & $2500 \mathrm{um} / 115 \mathrm{um}$ & $7500 \mathrm{um} / 110 \mathrm{um}$ \\
\hline M4(W1) & $2500 \mathrm{um} / 115 \mathrm{um}$ & $2500 \mathrm{um} / 110 \mathrm{um}$ \\
\hline M5(W10) & $25000 \mathrm{um} / 115 \mathrm{um}$ & $25000 \mathrm{um} / 110 \mathrm{um}$ \\
\hline M6(W10) & $25000 \mathrm{um} / 115 \mathrm{um}$ & $25000 \mathrm{um} / 110 \mathrm{um}$ \\
\hline M7(W2-A1,W3-A2) & $5000 \mathrm{um} / 115 \mathrm{um}$ & $7500 \mathrm{um} / 110 \mathrm{um}$ \\
\hline T8(W2-A1,W3-A2) & $5000 \mathrm{um} / 115 \mathrm{um}$ & $7500 \mathrm{um} / 110 \mathrm{um}$ \\
\hline T9(W3-A1,W5-A2) & $7500 \mathrm{um} / 115 \mathrm{um}$ & $12500 \mathrm{um} / 110 \mathrm{um}$ \\
\hline \hline
\end{tabular}

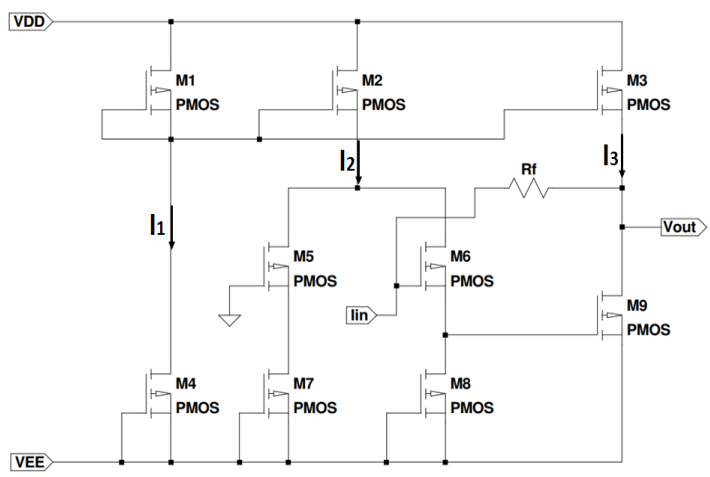

Fig. 3. A transimpedance amplifier design using flexible P-type OTFTs.

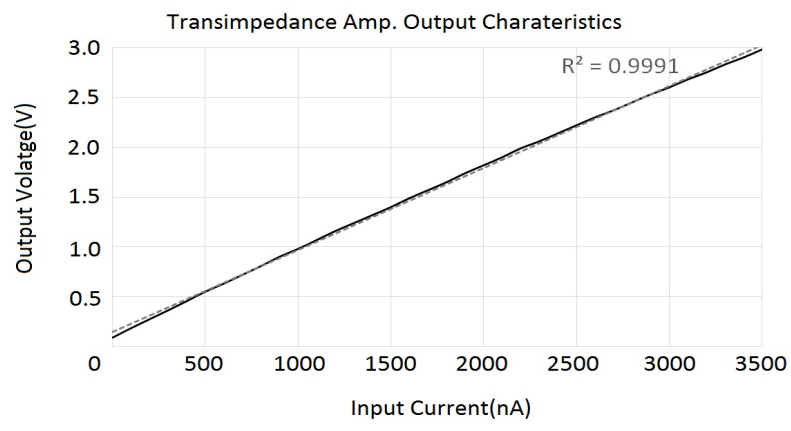

Fig. 4. Output voltage as a function of the input current levels obtained for the developed OTFT-based transimpedance amplifier.

By its turn, Fig. 4 shows the transimpedance amplifier's input current and output voltage characteristics, achieving a linearity correlation of 0.9991 in the region for data signal collection within a $3 \mathrm{~V}$ range. An $80 \mathrm{mV}$ drift is still obtained at the output of the amplifier without input currents, which can be compensated for inside the $3.3 \mathrm{~V}$ digitalization system connected to the amplifier 
(buffer) and explained in section D.

An improved version of the transimpedance amplifier (version 2) employed for $\mathrm{pH}$ sensing was recently manufactured and presented in [24] using the same technology from our team, but with reduced physical dimensions from $10 \times 10 \mathrm{~cm}^{2}$ to $3 \times 4 \mathrm{~cm}^{2}$, as depicted in Fig. 5. The circuit design principle employed is the same as described previously, with modifications on the W/L ratios of the transistors in order to provide signal conditioning enhancement for the $\mathrm{pH}$-type chemical sensor.

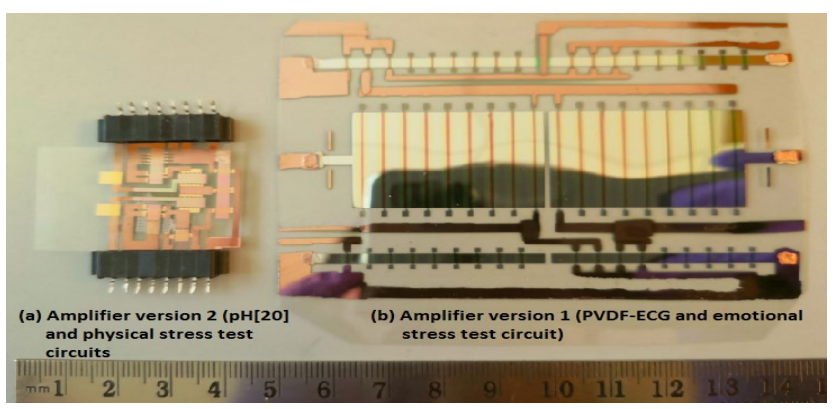

Fig. 5. (a) Amplifier version 2 for $\mathrm{pH}[20]$ and physical stress test circuits (b) Amplifier version 1 for PVDF-HRV and emotional stress test circuitry.

\section{B. PVDF-based mechanical deformation sensor and amplifier readout}

A PVDF sensor normally provides charge or voltage variations at its output when mechanically strained; being useful to detect motion or vibration from objects. In this experiment, we chose an off-the shelf capacitive type flexible piezoelectric sensor (LDT1028K PIEZO SENSOR, TE connectivity) as the artery pulse detector. As depicted in Fig. 6, the output of the PVDF sensor is connected to the inverting input of the developed OTFT-based amplifier for current-to-voltage conversion (I-V) and amplification, where the I-V feedback gain is set by the resistance of $\operatorname{Rf}(620 \mathrm{k} \Omega)$. By its turn, the non-inverting input of the amplifier is grounded to ensure common potential between input terminals, thus reducing the noise level in the circuitry. Output voltages are current changes from the PVDF sensor (Ip) when measuring the artery pulses and multiplied by Rf in accordance to Eq. 1.

$\mathrm{Vo}=-\mathrm{Rf} * \mathrm{Ip}$

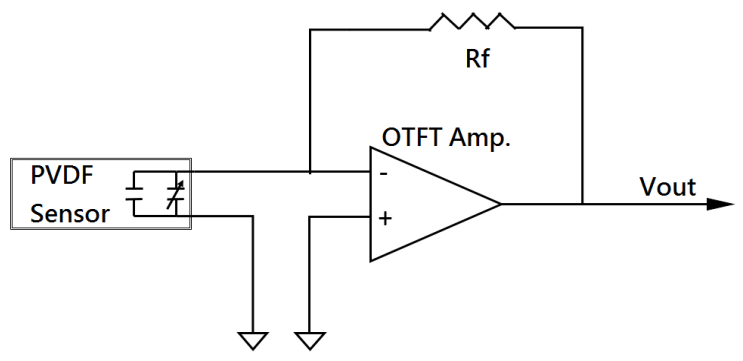

Fig. 6. Transimpedance amplifier interfacing directly the PVDF sensor at its inverting input terminal.

\section{Flexible pH-type sensor deposition and data readout}

Prior to the integration of the OTFT-based transimpedance amplifier with $\mathrm{pH}$ sensor deposition over a common substrate, five pairs of gold electrodes ( $99 \%$ purity, thickness of $50 \mathrm{~nm}$ ) were evaporated using the same process of manufacturing OTFTs and PET $(125 \mu \mathrm{m})$ substrate. Then, pH-type sensors were electrochemically deposited on top of the pre- treated gold surface. The initial cleaning procedure involved cyclic voltammetry using $50 \mathrm{mM} \mathrm{H}_{2} \mathrm{SO}_{4}$ at $50 \mathrm{mV} / \mathrm{s}$ for $100 \mathrm{cls}$. An additional layer of gold nanoparticles was also deposited to increase the surface area. These nanoparticles were deposited by reducing $0.3 \mathrm{mM} \mathrm{HAuCl}_{4}$ solution at a constant potential of $-0.2 \mathrm{~V}$ and the IrOx-based $\mathrm{pH}$ sensing membrane was deposited electrochemically by the method described by the authors of [15]. Fig. 7 shows (a) the calibration curves measured from an electrochemical station (CHI600E, $\mathrm{CH}$ instruments) and real-time solution testing (b) with the proposed NFC system, when interfacing a commercially available and ultralow input bias current amplifier (LMP7721, Texas Instruments) to cope with the high output impedance of the flexible pH-type sensor. The results obtained have showed very stable, repeatable and fast response times (less than $20 \mathrm{sec}$ ) for the sensor, with sensitivity reaching a level of $62.8 \mathrm{mV}$ per unit $\mathrm{pH}$. 

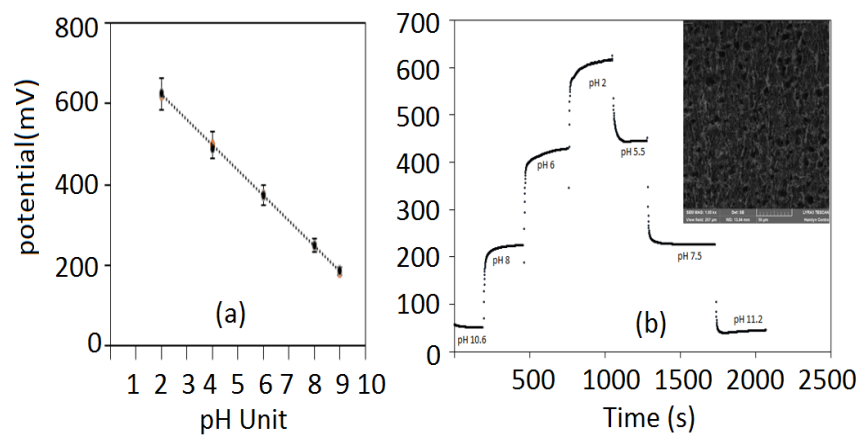

Fig. 7. (a) Calibration curves for $\mathrm{pH}$ sensors (with each point showing the mean and standard deviation) and (b) an example of real time data of the changes of $\mathrm{pH}$, inset shows the membrane of the flexible $\mathrm{pH}$ sensor.

The aforementioned impedance matching problem found between the $\mathrm{pH}$-type sensor and the flexible/rigid readout electronics was recently solved in [24] and implemented in this paper as well. In order not to compromise the circuit's design and topology, the same off-the-shelf buffer amplifier used during the chemical testing of the $\mathrm{pH}$-type sensor was inserted into the proposed system and connected in series to a resistor Ri of $1 \mathrm{k} \Omega$, as shown in Fig. 8. This resistor converts the voltages generated by the $\mathrm{pH}$ solution level into currents that are more suitable for being detected by a transimpedance-type amplifier (amplifier 2), with gain resistor set to $820 \mathrm{k} \Omega$ and bias level of $0.8 \mathrm{~V}$ applied directly to the reference electrode.

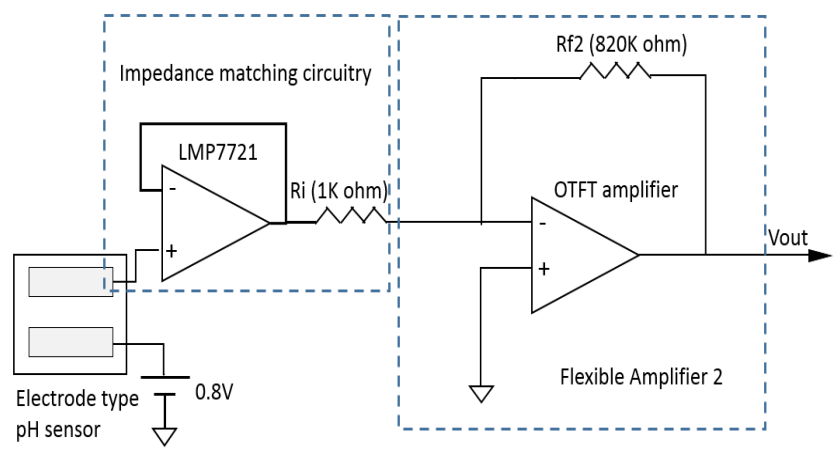

Fig. 8. Electronic circuit involved in $\mathrm{pH}$ sensing.

\section{Wireless back-end system and data transmission}

A 3.3V digitalization system made of off-the-shelf components only was designed to acquire the signals originating at the OTFTbased amplifiers with two input channels, one for the artery pulse detection and the other for $\mathrm{pH}$ measurements (vide Fig. 9). In both cases, a buffer amplifier (AD8506, Analog Devices) interfaces directly the signals from the OTFT-based amplifiers so as to accommodate any impedance mismatches verified between these signals and the analog-to-digital converter (ADC) located inside the microcontroller or MCU (PIC18F26J53, Microchip). The digitalization rate for the artery pulse signal was set to 100 samples per second (SPS) whereas, for the $\mathrm{pH}$ signal, a rate of 10 SPS was used with 12-bit of resolution employed for both signals. The transmission of the digital samples can be performed in two different modes, by Universal Serial Bus (USB) to a remote computer, or NFC through a mobile phone. In the former, a signal converter (FT234, FTDI Chip) converts the acquired samples from the Universal Asynchronous Receiver-Transmitter interface (UART) of the MCU into USB signals in real-time, with transmission rates of $460.8 \mathrm{kbps}$. For NFC, a tag was embedded within the device (NT3H1101, NXP) that can hold up to $1 \mathrm{kB}$ of data directly accessible by the external radio frequency (RF) field generated by the mobile phone. Data is transferred every 5 seconds to the tag through Inter-Integrated Circuit interface (I2C) at a rate of $9600 \mathrm{bps}$, which can then be visualized inside a mobile phone's app developed in Android Studio by swiping the phone over the printed NFC coil included in the digitalization system. This coil was designed with $0.4 \mathrm{~mm}$ (width) copper tracks and total number of turns equal to 6 , occupying an area of $3 \mathrm{~cm} \mathrm{x} 4 \mathrm{~cm}$.

The power supply is provided by the USB $(5 \mathrm{~V})$ when the system is connected directly to a computer or by a rechargeable lithium polymer battery (LP-402025-IS-3, BAK) for portable NFC recordings. With a total capacity of $165 \mathrm{mAh}$, the battery can power continuously the system for periods of 8 hours, as the total current consumption from electronics is around $20 \mathrm{~mA}$. The battery can also be recharged through USB since a Li-Ion/Li-Polymer charge controller is provided within the system (MCP73812T, Microchip) and down-converted to a level of 3.3 V by DC/DC converter (TC1015-3.3V, Microchip).

Finally, a $10^{\text {th }}$-order low-pass filter with cut-off frequency of $20 \mathrm{~Hz}$ is implemented on the app and/or Matlab scripts to preprocess the artery pulse samples, in order to remove electromagnetic interferences originating mainly from the power grid line. The $\mathrm{pH}$ samples are also processed with a 6-point moving average filter to smooth signal transients produced by slowly varying temporal changes. 


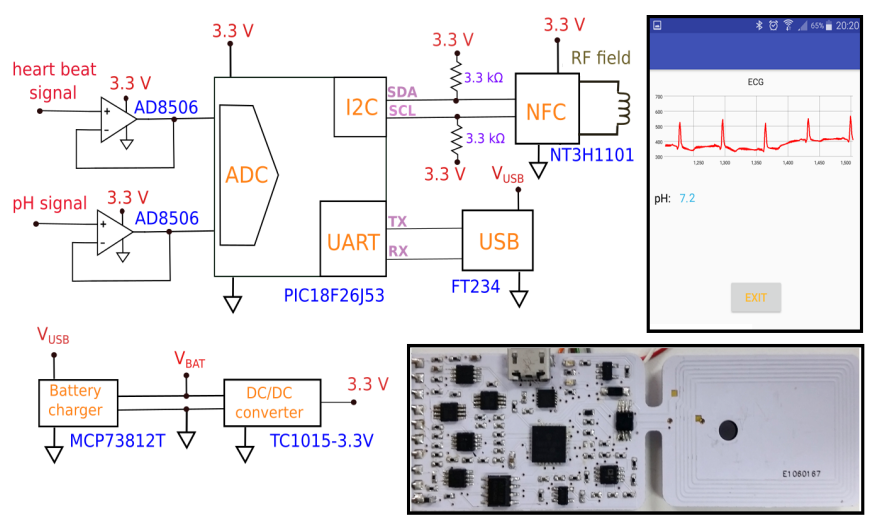

Fig. 9. Digitalization system developed for interfacing the OTFT-based amplifiers with USB and NFC connectivity: electronic schematic (left), mobile phone app (top right) and printed circuit board with components assembled on the top layer only (bottom right).

E. Flexible sensors and circuits comparison

A comparison table of flexible sensors and signal conditioning circuits' solutions between the proposed system and [9-10] is shown in table II.

TABLE II

PROPOSED SENSORS AND AMPLIFIERS SOLUTIONS VS. [12-13]

\begin{tabular}{|c|c|c|c|}
\hline $\begin{array}{l}\text { Flexible } \\
\text { sensors and } \\
\text { circuits }\end{array}$ & $\begin{array}{l}\text { Proposed } \\
\text { flexible } \\
\text { system. }\end{array}$ & $\begin{array}{l}\text { Sekine } \text { et al. } \\
\quad \text { [13] }\end{array}$ & $\begin{array}{c}\text { Yoon et al. } \\
\text { [14] }\end{array}$ \\
\hline PVDF & $\begin{array}{l}0.016 \mathrm{~V} \\
\text { (Piezoelectric } \\
\text { film sensor } \\
\text { model: LDT1- } \\
028 \mathrm{~K} \text { ) }\end{array}$ & $\begin{array}{l}0.008 \text { V(WRIST) } \\
0.04 \text { (NECK) }\end{array}$ & $35 \mathrm{uV} / \mathrm{mmHg}$ \\
\hline $\mathrm{pH}$ & $\begin{array}{l}62.8 \mathrm{mV} / \mathrm{per} \\
\mathrm{pH}\end{array}$ & Not applicable & Not applicable \\
\hline $\begin{array}{l}\text { Amplification } \\
\text { stage circuits }\end{array}$ & $\begin{array}{l}\text { Truly flexible } \\
\text { transimpedance } \\
\text { amplifiers with } \\
\text { gains set up by } \\
\text { Rf and Rf2 }\end{array}$ & $\begin{array}{l}\text { Av=11 with an } \\
\text { off-the shelf } \\
\text { amplifier on } \\
\text { flexible } \\
\text { substrate }\end{array}$ & Not applicable \\
\hline
\end{tabular}

\section{F. Algorithm for R-peak estimation from artery pulses}

A computer based algorithm implemented in Matlab (MathWorks, MA, USA) was used to process the artery pulse data that was acquired directly from healthy subjects, wearing the novel flexible front-end electronics and digitalization system. Compared to a traditional two leads or three leads electrode-based ECG measurement system, the vital signals were directly over the common carotid artery. A flowchart of the processing routines implemented on the computational side is shown in Fig. 10, whereas Fig. 11 exhibits a five second long dataset generated by one male subject during the testing of the proposed device and algorithm. 


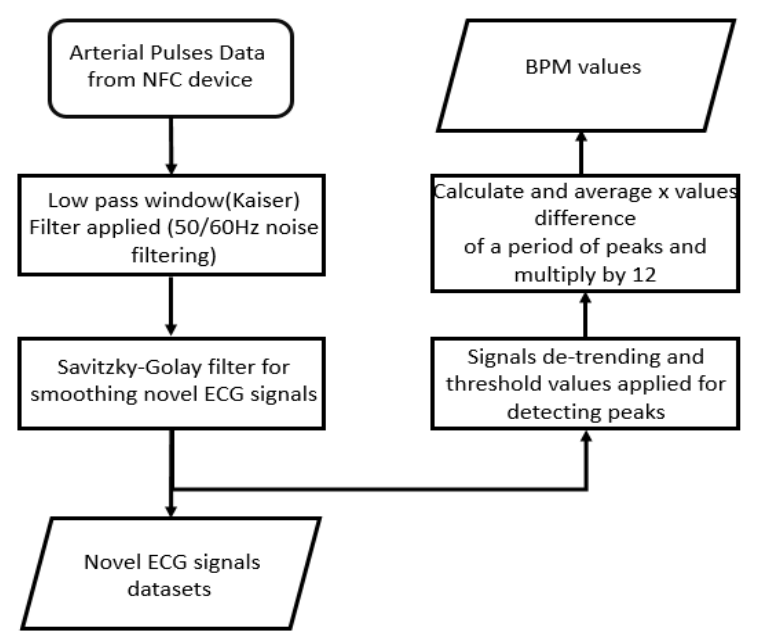

Fig. 10. A flowchart of Matlab algorithm for processing and detecting artery pulses ("ECG") signal from the novel flexible front-end electronics using a PVDF sensor as the signal transductor.
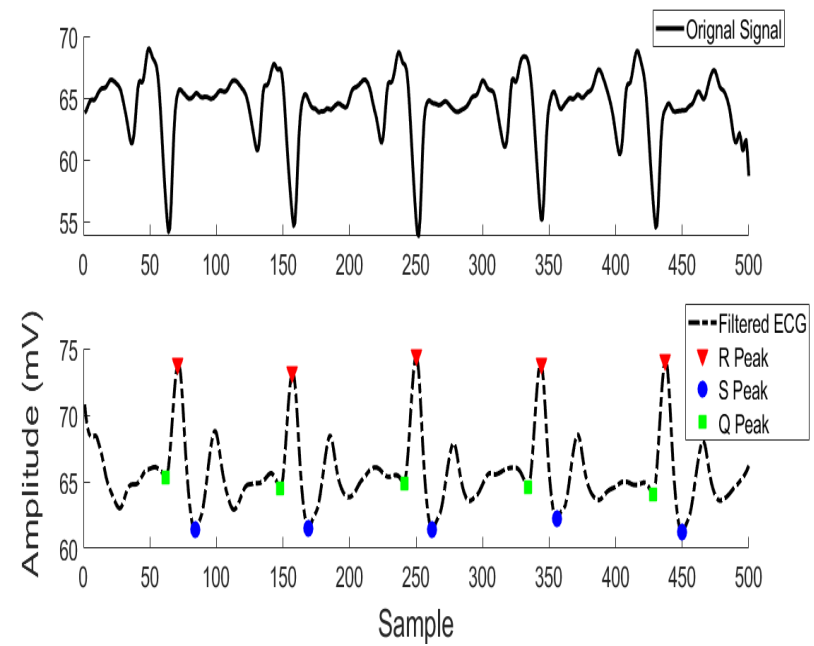

Fig. 11. Top: artery pulses obtained after 50/60HZ filtering; Bottom: ECG QRS complex waveform obtained and signal processed from one healthy subject.

As ECG measures electrical potential depolarization over the heart and our proposed system the pressure wave generated at a close distance from the heart, it is plausible to assume that the main features depicted in the previous figure are synchronized with the main cardiac events recorded by ECG, such as the QRS complex, thus enabling the location of the R peak over the artery pulse signal. To further corroborate this hypothesis, which impacts severely the determination of the heart rate (HR), the proposed system was verified against a commercial and portable ECG recording machine (EMAY-PM10, CE certificated), obtaining an error rate less than $+/-1$ beat per minute for a heathy subject, as shown in Fig. 12 for a temporally localized segment. Due to the output data format provided by the EMAY PM10 machine, we can only validate heart rate change by manual inspection and, therefore, more detailed statistical analysis in terms of data validation between the two devices cannot be presented in this paper.

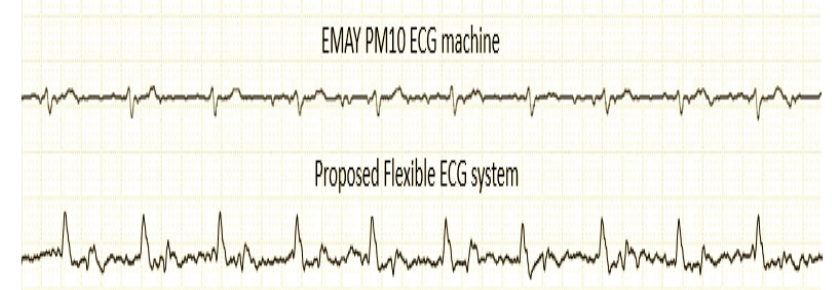


Fig. 12. Heart rate validation using the portable EMAY PM10 ECG machine and the proposed system using the USB interface for data streaming.

\section{EMOTIONAL AND PHYSICAL STRESS ENVIRONMENT TEST PROTOCOLS AND HEALTHY SUBJECTS’ TRAIL}

A research study of emotional stress level was carried out to understand how cardiac coherence fluctuates when participants are playing violent video games [4]. The results obtained by the authors of this study have shown a lower HRV (or higher HR) in the group of participants playing the violent video in comparison to the group playing non-violent games, whose HRV remained at the same level. Other studies were conducted [5-6] to understand how fear-induced stress media could affect vascular response in humans. The results obtained by the authors of the study have shown that both artery pressures and HR increased at the forearm limb and finger of the tested subjects, in the locations where ECG, blood pressure and blood flow Doppler devices were previously placed.

Within the present paper, we developed a test protocol (protocol-1) composed by a series of media film clips merged together into a movie with total duration of $7.5 \mathrm{~min}$ and to be displayed to willing volunteers in order to understand the relationship between the induced stress and HR levels. The movie was divided into specific temporal segments, with the first 60 seconds showing a still picture in the background accompanied by a peaceful church song, followed by five 60 -seconds long segments with increasing level in the content of violent scenes, before a single minute clip with kidnapping scenes. The movie was then finalized by a one minute clip showing an animated film with some relaxing song. Ten healthy subjects (male: $80 \%$, female: $20 \%$; ages from 25-35 years old; body mass index - BMI - from 20 to 26) were recruited under the ICREC ethical approval reference 18IC4816 and with no reported medication being taken before and during the day of the experiment. All healthy subjects were asked to sit on a chair with the PVDF sensor placed on the neck and covered by a biocompatible textile tape (KT tape) to sense the artery pulses during the movie, as shown in Fig. 13a. For the second session of the trial (protocol-2), three of the volunteers (males only; ages from 2630 years old; BMI of 20-24) were asked to cycle gently on an excise bike (Trainer 2013, Wattbike) with resistance adjusted to level 5, during an initial period of five minutes (warm-up stage), followed by a speed-up stage with fast cycling for an additional period of five minutes. After the ten minute period for exercise, subjects were asked to stop cycling and just sit comfortably on the bike for another period of 10 minutes (rest stage), as displayed in Fig. 13b. Simultaneous HR and pH data collection was only performed during the second session of the trial by placing a previously calibrated $\mathrm{pH}$-type sensor on the back of the volunteers (Fig. 13c). Additional $\mathrm{pH}$ level assessment was performed in situ by a $\mathrm{pH}$ meter (pH Tester, Hanna GroLine) in the sweat produced by the back of the body, after the cycling exercise was finalized.

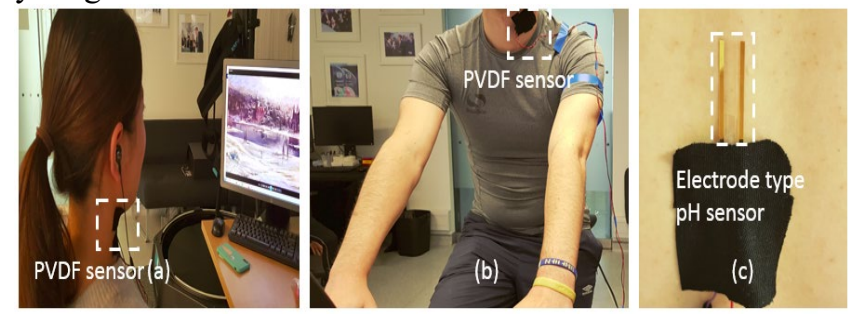

Fig. 13. (a) Photo exhibiting one volunteer during the test protocol 1 (emotional stress) with PVDF sensor placed on the neck and covered by a biocompatible tape (b) Physical stress monitoring (protocol 2) with PVDF sensor placement (c) pH-type sensor placed on the back of the volunteer performing protocol 2 .

In terms of results obtained during the trials, Fig. 14 exhibits three sets of data captured from the 7.5 min emotional stress monitoring for one of the volunteers. The HR registered during the first minute was 65 , increasing to 75 during the period of watching violent scenes. There were no significant HR changes in the last minute of the movie, which can indicate that the subject's mood is still affected by the previous violent scenes, with no recovery in the time window provided for the experiment. 

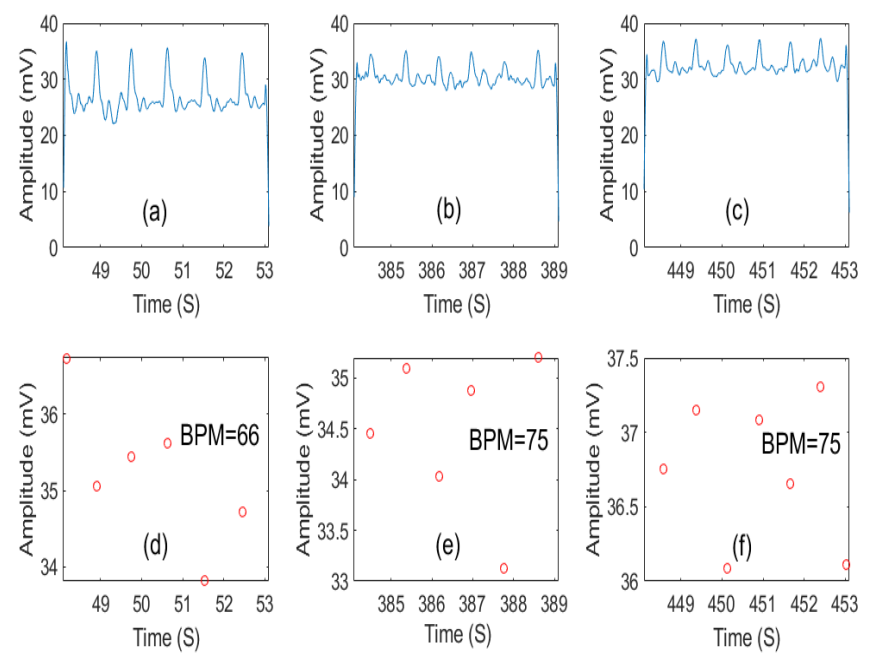

Fig. 14. (a) HR from an individual obtained when watching a still picture and listening to a peaceful church song (b) HR during the exhibition of the violent scenes from the movie (c) HR at the final of the movie, composed by animated scenes with relaxing song. (d) Peaks detection and BPM for status (a), (e) peaks detection and BPM for status (b), (f) peaks detection and BPM for (c).

With the same volunteer, a similarity comparison in terms of the estimated HR level was performed between a high performance data acquisition device (DT9834, sampling rate of 100 SPS) and the proposed system, using the NFC interface for data collection in simultaneous, as depicted in Fig. 15. Both datasets were then post-processed using the R-peak estimation routine from the detection algorithm, with a similar HR signal trend being obtained between the two different systems.

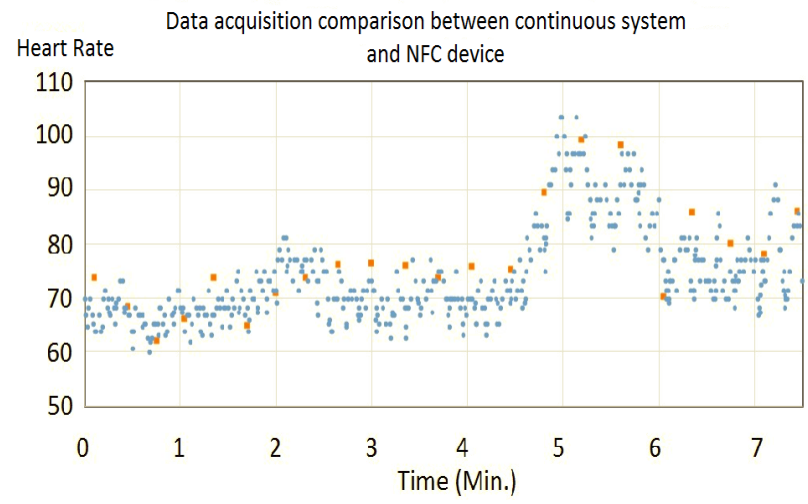

Fig. 15. Data acquisition comparison between a high performance acquisition system (blue dots) and proposed NFC device (orange dots), with HR level estimation performed by the detection algorithm (single volunteer).

Detailed results obtained for the ten subjects performing protocol-1 are presented in Fig. 16. The HR were calculated and presented as percentage of the initial artery pulse detected at the beginning of the movie (phase a). The temporal stamps for the violent scenes are grouped in rectangles over the graph area and corresponding to different phases of the movies: $b$ - shooting in a public area (1 minute long); $\mathrm{c}$ - stabbing scenes with screaming (2.5 min); $\mathrm{d}$ - stabbing scenes without screaming (1 min); e kidnapping scene (1 min). An average of the HR level obtained from the five most standard data subjects was also calculated and presented in the graph (solid line). 


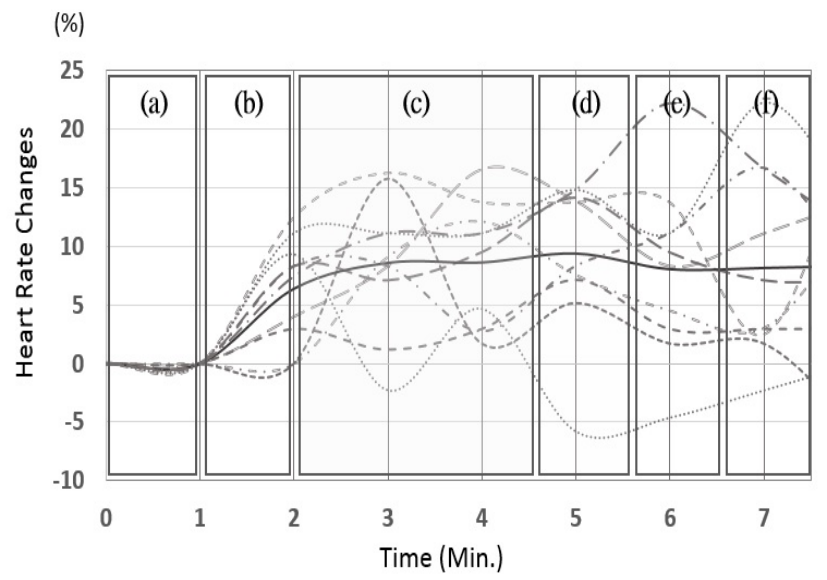

Fig. 16. HR variations (dotted lines) recorded for the ten healthy volunteers during the movie and presented in terms of the percentage deviation registered at the beginning of protocol-1. Each rectangle over the graph reflects different scene contents within the movie: a - still picture with peaceful song played in the background; $b$ - chip with shooting scenes (violent); $\mathrm{c}$ - clip with stabbing scenes and screaming (violent); $\mathrm{d}$ - clip with stabbing scenes and no screaming (violent); e - kidnapping clip; $\mathrm{f}$ - animated clip with a final relaxing song. The black solid line corresponds to the average HR calculated from the five most standard data subjects that performed the trial.

In order to realize the design concept of integrating two different sensors for stress detecting and obtain $\mathrm{pH}$ level data in the amount of sweat and within a short period of time, a second test protocol (protocol-2) for physical stress monitoring was also conducted in three healthy volunteers. HR and $\mathrm{pH}$ results are presented in Fig. 17 during the period of conducting the trial involving exercise activity only. From the results, the more significant changes to HR occurs during the period of exercise (first ten minutes), with gradual decrease on the level during the rest stage (last 10minutes). It is also verified that, for subject three, the rise in HR coincided after the the beginning of the speed-up stage (at minute 5) whereas, for the other subjects, this rise in HR started within the warm-up stage (roughly at minute 2). The different body composition and level of physical fit amongst the subjects may explain this phenomenon. Aslo the return to pre-exercise HR levels is accomplished more rapidly for subject 3 , in opposition to subject 1 where the HR did not return to the rest condition during the time window provided for the exercise.
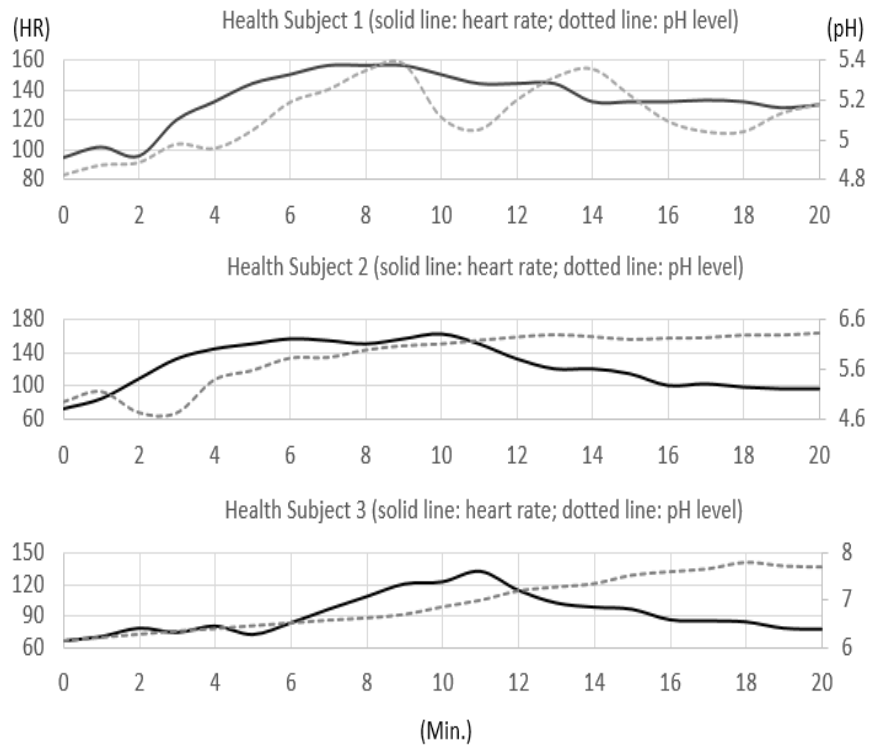

Fig. 17. Results obtained for the three healthy subjects performing protocol-2. Subjects' data are shown from top to bottom: Solid line - heart rate; dotted line - $\mathrm{pH}$ level.

Finally, regarding the sweat $\mathrm{pH}$ levels recorded for the three subjects, we found a similar trend in the evolution of the $\mathrm{pH}$ curves as obtained in [15], with similar protocols involved. The gradual rise in the $\mathrm{pH}$ level due the exercise is a consequence of the release of more and more sweat-containing $\mathrm{H}^{+}$protons, which forces a shift in the level of skin $\mathrm{pH}$ towards a more alkaline condition. The non-recovery of the pre-exercise $\mathrm{pH}$ level after the end of the trial for subjects 2 and 3, suggests that the sweat accumulating 
around the sensor leads to its saturation and a mechanism to ensure cyclic reflow of sweat around the area must be accounted for in a future re-design of the system, in the form of microfluidic channels and/or another type of sweat absorbent techniques.

\section{CONCLUSION AND FUTURE WORK}

A novel OTFT-based transimpedance amplifier was designed using an engineering prototype sheet which was manufactured by R2R printing technology and presented in this manuscript. These amplifiers were developed to amplify the ECG-related signals detected by a single commercially available mechanical deformation PVDF sensor and sweat $\mathrm{pH}$ level detected by sensors developed in-house. A low powered NFC device was connected to the outputs of the amplifiers for continuous monitoring of artery pulses and $\mathrm{pH}$ levels from healthy subjects. An algorithm to extract the artery pulse's main component was also presented in order to relate it with the R-peak commonly found in the normal ECG wave, with validation by trend comparison with commercial ECG equipment. Regarding the human trials, the average changes in HR measured from subjects using the proposed system and protocol (protocol-1) also confirmed the results reported in [4-6] for emotional stress induction based on media film clips. For the chemical sensing, the common PEN flexible pH-type sensor developed in house revealed a sensitivity of $62.8 \mathrm{mV}$ per unit of $\mathrm{pH}$, which is comparable to the standard value for $\mathrm{pH}$-proton potential generation in solution $(\simeq 50 \mathrm{mV} / \mathrm{unit})$ [25]. Therefore, a second test protocol (protocol-2) for physical stress monitoring was also conducted with reduced number of volunteers to test the feasibility of the proposed system and method to detect both HR and sweat $\mathrm{pH}$ during exercise.

To the best knowledge of the authors, this is the first study combing physical (piezo) and chemical (pH) sensors with OTFT integrated circuitry, with application in the assessment of stressful events, lacking only the confirmation of the $\mathrm{pH}$ signal induced variation induced by psychological (mental) stress. In the future, we hope to finalize integrating the wireless telemetry onto the same flexible and bendable substrate through a combination of manufacturing processes such as R2R and 3D printing, thereby making the single-piece device easier to wear over the body in the context of personal (exercise) or medical mental health monitoring. In this latter scenario, the availability of flexible devices structurally conformal to the body shape and contour is of paramount importance to help minimizing not only the discomfort felt by mental health patients during long-term monitoring, but also the individual and social stigmas associated with the use of medical devices.

It is the belief of the authors that chemical information (e.g. pH level) is important in any mental health-related condition, with tendency to produce waveforms similar to the ones observed in a typical GSR signal, whose faster signal transients (fluctuations) have already been correlated with the immediate response of the autonomous nervous system to psychological stress and the lower frequency content of the GSR signal to a continuous exposition of a particular stressor (e.g. demanding physical/mental task). Finally, only a multi-modal sensing device with direct physiological signal monitoring such as the one presented in the current manuscript can effectively contribute to differentiate between the aforementioned physical and psychological stressors (type and intensity) whenever they occur, which can ultimately guide the design of novel algorithms for detection of individual stress levels more accurately than the ones already implemented inside some mobile phone's apps and computer programs.

\section{ACKNOWLEDGEMENT}

The project is funded by EPSRC under the project grants: EP/M015173/1 (University of Oxford) and EP/L014149 and EP/N027132/1 (Imperial College London). Authors would like to acknowledge the support from the Department of Engineering Science, Department of Materials (University of Oxford) and the Hamlyn Centre (Imperial College London). Authors would also like to personally thank Prof. Steve Collins for the technical advice for the characterization of OTFT devices. Finally, the authors would like to express their gratitude to the Newton Fund's 2018 Researcher Links Workshop in Mental Health Technology for making the collaboration between two universities possible.

\section{REFERENCES}

[1] E. Charmandari, C. Tsigos and G. Chrousos' Endocrinology of The Stress Response'Annual Review of Physiology,vol.67, pp. 259-284, 2005

[2] K. M. Conley and B. J. Lehman,' Test Anxiety and Cardiovascular Responses to Daily Academic Stressors', Stress Health, vol.28(1), pp.41-50,2012

[3] B. Sandin and P. Chorot,' Changes in Skin, Salivary, nad Urinary pH as Indicatos of Anxiety Level in Humans', Psychophysiology, vol. 22, no. 2, pp. 226-230, 1985

[4] Y. Hasen, L. Begue and B. J. Bushman,'Biolent Video Games Stress Prople Out and Make Them More Aggressive',Aggressive Behavior, vol.39, pp. 64-70, 2013

[5] N. Hayashi, N. Someya, T. Maruyama, Y. Hirooka, M. Yamaoka Endo and Y. Fukuba,' Vascular responses to fear-induced stress in humans', Physiology \& Behavior vol.98,pp. 441-446, 2009

[6] S. D. Kreibig, F. H. Wilhelm, W. T. Roth and J. J. Gross,' Cardiovascular, electrodermal, and respiratory response patterns to fear-and sadness-inducing films', Psychophysiology, vol. 44, pp.787-806, 2007

[7] B.M.G. Rosa and G.Z. Yang, "A Flexible Wearable Device for Measurement of Cardiac, Electrodermal, and Motion Parameters in Mental Healthcare Applications", IEEE Journal of Mental Healthcare Applications, vol. 23, pp 2276-2285, 2019.

G.Z. Yang, "Body sensor networks", Springer, 2006

[9] A. Muaremi, B. Arnrich and G. Troster, "Towards Measuring Stress with Smartphone and Wearable Devices During Workday and Sleep" BioNanoScience, vol. 3, pp 172-183, 2013.

[10] J. M. Peake, G. Kerr and J.P sullivan, "A critical review of consumer wearables, mobile applications, and equipment for providing Biofeedback, monitoring stress, and sleep in physically active populations" Front Physiol. Vol 8, pp743(1-19), 2018

[11] W.Gao et al "Fully integrated wearable sensor arrays for multiplexed in situ perspiration analysis." Nature, vol. 529, pp. 509-514, 2016 
[12] G.Z. Yang, ' Sensor embodment and Flexible electronics', in Implantable Sensors and System Springer,pp.197-279, 2018

[13] T. Sekine, R. Sugano, T. Tashiro, J. Sato, Y. Takeda, H. Matsui, D. Kumaki, F. Domingues Dos Santos, A. Miyabo and S. Tokito,' Fully Printed Wearable Vital Sensor ofr Human Pulse Rate Monitoring using Ferroelectric Polymer', Scientific Report, vol.8, pp.1-10, 2018

[14] S. Yoon, J. K. Sim and Y-H Cho,' A Flexible and Wearable Human Stress Monitoring Patch', Scientific Report, vol. 6, pp.1-11, 2016

[15] S. Anastasova, B. Crewther, P. Bembnowicz, V. Curto, H. M.D. Ip., B. Rosa and G.Z. Yang,' A wearable multisensing patch for continuous sweat monitoring', Biosensors and Bioelectronics, vol. 93, pp.139-145, 2017

[16] A. J. Bandodkar, V. W. Hung, W. Jia, G. Valdes-Ramirez, J. R. Windmiller, A. G. Martinez, J. Ramirez, G. Chan, K. Kerman and J. Wang,'Tattoo-based potentiometric ion-selective sensors for epidermanl pH monitoring', Analyst, vol. 138, pp. 123-128, 2013

[17] M. Berthelot, C.M. Chen, G.Z. Yang and B. Lo,' Wireless wearable self-calibrated sensor for perfusion assessment of myocutaneous tissue', IEEE 13 ${ }^{\text {th }}$ International Conference on Wearable and Implantable Body Sensor Network, San Francisco, USA, pp.171-176, 2016

[18] B. M. G. Rosa, and G. Z. Yang,' Smart wireless headphone for cardiovascular and stress moniotoring', IEEE $14^{\text {th }}$ International Conference on Wearable and Implantable Body Sensor Networks, Eindhoven, Netherlands, pp. 75-78, 2017

[19] T Cosnahan, A.A.R.Watt and H.E. Assender 'Flexography printing for Organic Thin Film Transistors' Materials Today: Proceedings 5,pp.1605116057,2018

[20] G.A.Abbas, Z. Ding, H.E.Assender, J.J Morrison, S.G. Yeates, E.R. Patchett \& D.M. Taylor 'A high-yielding evaporation-based process for organic transistors based on the semiconductor DNTT' Org. Elec. Vol. 15 pp.1998-2006,2014

[21] D. M. Taylor,' Vacuum-thermal-evaporation: the route for roll-to-roll production of large-area organic electronic circuits', Semicondcutor Science and Technology, vol. 30, pp.1-14, 2015

[22] N. K. Za'aba, J. J. Morrison, and D. M. Taylor,' Effect of relative humidity and temperature on the stability of DNTT transistors: A density of states investigation', Organic Electronics, vol. 45, pp. 174-181, 2017

[23] Z. Ding,' Large area vacuum farbrication of organic thin-film transistors', Ph.D Thesis, University of Oxford, 2014

[24] K. Zhang, C. M. Chen, S. Anastasova, B. Rosa, B. Lo and H. E. Assender,' Roll-to-Roll processable OTFT-based Amplifier and Application for pH sensing', The $16^{\text {th }}$ IEEE-EMBS International conference on Body Sensor Networks, 19-22 May, UoI, Chicago, USA 2019, pp.1-4

[25] B. Gil, S. Anastasova and G. Z. Yang, 'A Smart Wireless Ear-Worn Device for Cardiovascular and Sweat Parameter Monitoring During Physical Exercise: Design and Performance Results', Sensors, vol. 19, 2019. 